\title{
IoT based water reservoir monitoring system
}

\author{
Dr. S.R.Vijayalakshmi, Associate Professor, \\ Department of Electronics and communication systems, Sri Krishna Arts and Science College, Coimbatore, \\ Tamilnadu, India \\ vijayalakshmisr@skasc.ac.in
}

\begin{abstract}
Wireless sensor network makes the innovative capability of real-time communication anywhere anything at any time. Internet of Things produces numerous sensor devices connected to exchange the collected information and sensed data. Both the technology is involved in water's physical parameters quality monitoring. Water is stored in the tank, lakes, pool, dam, and water reservoir. Hence, water quality has to be checked before usage nowadays. This system checks the physical parameters of water for its quality identification to avoid improper unfit water consumption. The initial monitoring of water's physical property will be done by the system remotely in real-time and if needed their chemical properties done at laboratories. A laboratory check is recommended after analyzing the physical quality of water. This system is cost-effective and efficient designed to monitor the water's physical property. This paper discusses hardware modules and software blocks for performing physical characteristics of water. In this paper, the monitoring system consists of several sensors to determine the physical qualities of water in the reservoir such as the potential of hydrogen $(\mathrm{pH})$, temperature, humidity, electrical conduction, turbidness, Total Dissolved Solvents (TDS) of the water. The Microcontroller unit is interfaced with these sensors along with a wi-fi communication module. The collected data is sent to the cloud platform. All information is visible to the end-user at the instant of measurement. If the physical parameters are not within the standard range, the water is recommended to check its biochemical parameters at the laboratory for further quality identification. This paper gives an idea about the method of quality checking of water using IoT and WSN technology.
\end{abstract}

Keywords: Cloud platform, Internet of Things, Physical parameters of water, Potential of Hydrogen, Water quality monitoring.

\section{Introduction}

The traditional methods of checking water parameters are done manually. Water samples were obtained and sent to laboratories located somewhere. It is a time-consuming process, costlier, and needs human resources. It could not collect and process data in real-time. This monitoring system contains a micro controlling unit, all basic sensors, and a communication device. It is compact. It is very useful to measure $\mathrm{pH}$, turbidity, TDS, EC, Temperature, Humidity of the water continuously and in real-time. This real-time data is sent via a wireless communication module to the monitoring station. The objective of the project is to develop low cost, real-time, smartboard for monitoring water reservoirs and water bodies. This, IoT smart board is designed with sensors for water physical parameter measurements, microcontroller, ZigBee transceiver, and power supply.

\subsection{Literature survey}

Around 5 million people die due to waterborne illnesses around the globe [1]. Tradition water quality monitoring system involves the physical collection of water at different many locations, storing the collected samples in a central particular location, and exposing the samples to laboratory diagnostic testing which is tedious and time-consuming. The water values are measured by the system board smartly in real-time by the water board authorities, then it will be helpful for quality drinking water, standard maintenance, quality monitoring, and testing if required. Sung Min Cha at el. [2] analyzed the effect of ecological flow organization and defects in the environment due to water quality at Yongsan river. Jyotirmaya Ijaradar and Subhasish Chatterjee [3] discussed the water parameters measurement method at the time of monitoring. Sridharan, S [4] analyzed the water parameters measurement technique using a sensor networking wirelessly. Natalia and Malgorzata [5] discussed the biological 
methods used to assess surface water quality. Seema Tiwari [6] analyzed the water quality parameters. Real-time monitoring of water quality is an unsolved problem in water boards. Many exiting techniques are done using the water sample collected from different places and reviewing analyses which makes their application difficult in real-time monitoring and analysis of data. The disadvantages of the existing system are instrument fouling during real-time monitoring, loss of sensitivity, and frequent re-calibration. Population rises, industrial growth, urbanization are the causes for the deteriorating groundwater and drinking water quality. By monitoring the quality of drinking water, the government can ensure water is harmless to society. Hence, by monitoring the quality of water, people are protected from the diseases caused by polluted water. Accumulating pollution in water and water contamination in oceans, lakes, fish ponds, and river activates a universal call for extra innovative systems and technology implementation in the field of water quality monitoring systems by the water board. Currently, it is not followed frequently measurement of water quality at the water reservoirs. The values of water physical parameters determine the source of the good life for animals and humans. It gives good health for both if monitored. Else it is the source of sicknesses for humans and deaths of water animals. There is a need for the development of a measurement technique for wastewater monitoring that is online, smart, and non-invasive. This project aims to design of smart embedded system board for monitoring the quality of water and sewage water automatically.

\subsection{Background study}

In many locations, technical laboratory facility is not available for water quality checking. It needs a huge initial investment. Water analysis is done through the conventional method which follows the collection of water samples using testers. It never gives accurate results. Since the time of collecting the sample is important in some cases. Real-time monitoring is not possible with this technique. Need hours and days to complete the laboratory process. Unfortunately, water pollution is a major issue worldwide. Around $60 \%$ of total reserves of drinking water do not meet standards. This fact favors the proliferation of health issues in many cities and villages across the country. Hence, we have to ensure the safety of drinking water due to the excessive sources of pollutants. Singh et al., [7] discussed the system for smart bins located in various locations in cities for waste collection based on a decision system and dynamic modeling system for smart bin waste management. Roger [8] analyzed the communication protocols for smart sensors in IoT applications. Singh et al [9] discussed the digital temperature scanner using IoT. Thakur [10] analyzed Internet of Things (IoT) security in present status and future direction. All these papers discussed some of the IoT projects.

This paper is organized as follows. The first chapter is about the introduction and second chapter dealt with methodology and the third chapter is about the result and discussion and finally about the conclusion.

\section{Methodology}

The circuit design of the proposed system is made of physical sensing components, a micro controlling unit, a wireless transceiver chip, and a modified buoy for a water reservoir. The proposed sensor node detects physical properties such as water temperature, conductivity, total dissolved solvents, and $\mathrm{pH}$, etc. in a set time and compares with the standard value recommended by the water board. It displays the collected data in a pictorial representation and tabular formats through a personalized web-based page portal and pre-registered cellular phones apps to provide excellent service for end-users. Internet of Things and Wireless Sensor networking technologies are to be implemented for quality monitoring 
which ensures real-time ubiquitous data for water measurement. The water quality is maintained in lakes and water reservoirs. By using the proposed system, the government can provide quality water to the people and society.

Physical, chemical biological parameters of water to be measured for quality analysis. However, this system proposed measures only physical parameters of the water parameters using electronic sensors.

The parameters measured are

1. Water's $\mathrm{pH}$ value

2. Turbidity of the water

3. The temperature of the water

4. The humidity of the surrounding atmosphere

5. Electrical conductivity

6. Total dissolved solvents.

This IoT smart device can be functional in remote rivers, lakes, swimming pools, seaside areas, dams, buildings, small tanks, and other water reservoir bodies. The circuit hardware designed to monitor the water quality is made to work in ultra-low power mode. It is also designed for use in rugged environments. It could be placed in smart cities to access many locations to find the drinking water quality to avoid risk to public health. The Temperature, $\mathrm{pH}$, conductivity (salinity), turbidity with water leakage detector is interfaced to the MSP430 microcontroller. The sensor node algorithm is designed for calibration, measurement, water quality checking, comparing sensed values with standard values suggested by the water board, display of information, storage of data, and transmitting data to a server located in the water board.

Wireless sensor networks and the Internet of technology are selected for measuring physical water parameters in remote areas. The cost to manufacture this node is very low. It gives data in real-time. It reduces manual operations and does need not collect samples. Wireless sensor network to monitor water is implemented as three modules such as data collection, data storage, and data retrieval. System-oriented architecture for water IoT is applied with four layers (sensing, network, service, interface) for monitoring water information.

\subsection{Hardware unit for water sensor node}

The system designed uses sensors such as $\mathrm{pH}$ sensor, conductivity sensor, total dissolved solids sensor. These sensors are interfaced with the MCU MSP430 microcontroller. Zigbee module and wi-fi module are interfaced with MCU for transmitting the information to the monitoring station. The analog information of the sensors is converted into digital information for processing. The software algorithm receives signals from the monitoring station to measure parameters whenever necessary.

This signal is received by the transceiver Zigbee which is connected in the $\mathrm{I} 2 \mathrm{C}$ module. The received information is processed by the microcontroller and sent the activation signal to the sensor for measuring the water parameters to the sensor. The sensors are connected to the ADC module. This ADC module process the information and checks with the standard value and displays the information on the LCD module. The MCU sends the alert signal to a monitoring station for the measured parameters reception. After receiving an alert signal from the monitoring station, it is ready to receive 
the water's physical parameters. The block diagram of the hardware module is shown in figure 2.1 .

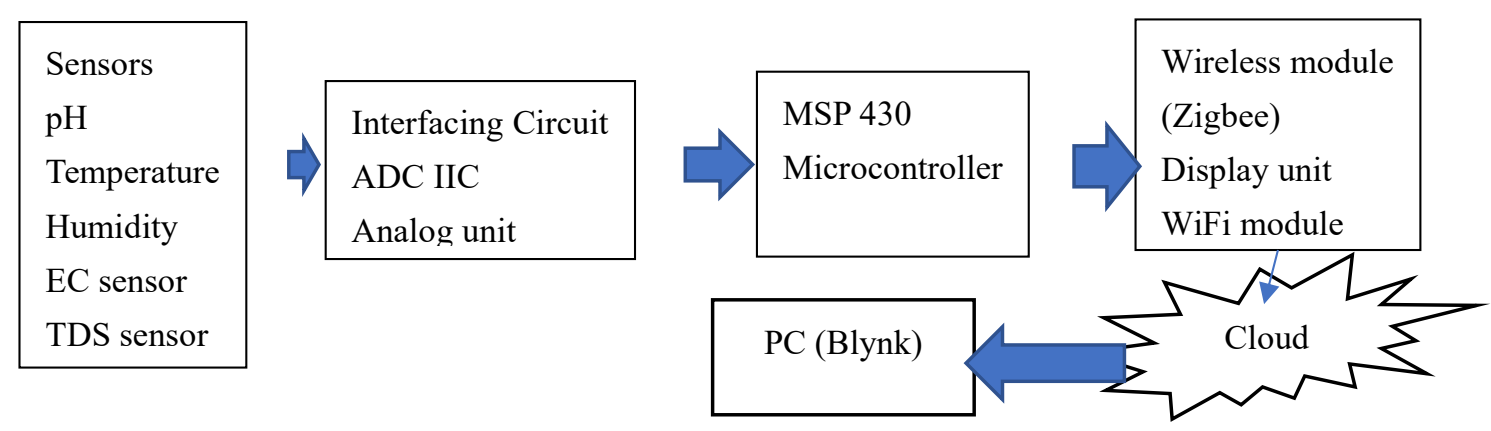

Figure 2.1. Block diagram of the hardware module

The sensor node is designed with Temperature (PT1000 sensor), $\mathrm{pH}$, conductivity (salinity), turbidity sensors. These sensing components are interfaced to the MSP430 micro controlling unit using their corresponding interfacing circuits. The sensor node collects physical water quality parameters and pre-process data from the sensors and transmits data to the server for water quality monitoring. The node is placed on the buoy which is made of a rubber tube. This rubber tube was covered with lightweight aluminum to float in water. The hardware electronic sensor board and battery (or solar panel) are situated on the upper part of the buoy. Solar power is used to ensure a continuous energy supply. The sensor node collects physical water quality parameters and processes data from the sensors. The sensor board sends data to the webserver and cloud platform for water quality monitoring.

\subsection{System design}

The system can measure the physical parameters of the water and stores data in a database. The information collected in real-time and timely information is given to the water board authorities and end-users of the system. The data are sent to the cloud for display purposes on the website. The data is sent to the user as SMS at the time of measurement.

The system at water board is designed to provide the following:

1) Data collection from different sensor nodes located in many locations

2) Storage of all water parameters in the database

3) Data propagation and display (charts, pictorial representation, tabular and data formats)

4) Low power supervision for longer measurement periods

5) Maintenance of standard water quality

6) Activity trigger for testing if not met quality

7) Data will be sent through the Internet for record

8) Sending data to the server or pre-identified cell phone number using wi-fi transceiver for long-distance

9) Zigbee for short distance or to another node communication

10)Web-based monitoring displays the output of water

The communication protocol between devices and servers and between servers and the cloud platform is cellular wi-fi. properties.

\subsection{Algorithm design for physical parameter sensors}


The below discusses the algorithm and flowchart for the water reservoir monitoring system. Flowchart 2.2 shows the flow of the information processing done by the water reservoir monitoring system board.

\section{Algorithm}

Step 1: start and send msg to collect data from sensors

Step 2: Read temperature, conductivity, $\mathrm{pH}$, turbidity, camera Image

Step 3: Print all sensed values

Step 4: Check for sensed value with standard value; Turbidity is less than 5; $\mathrm{pH}$ within 6.5 and 8.5; conductivity between 300 and 800 ;

Step 5: within limit print water quality good and use for drinking

Step 6: else water is bad and recommend for testing

Step 7: goto step 1 Parameters to be checked

Flowchart

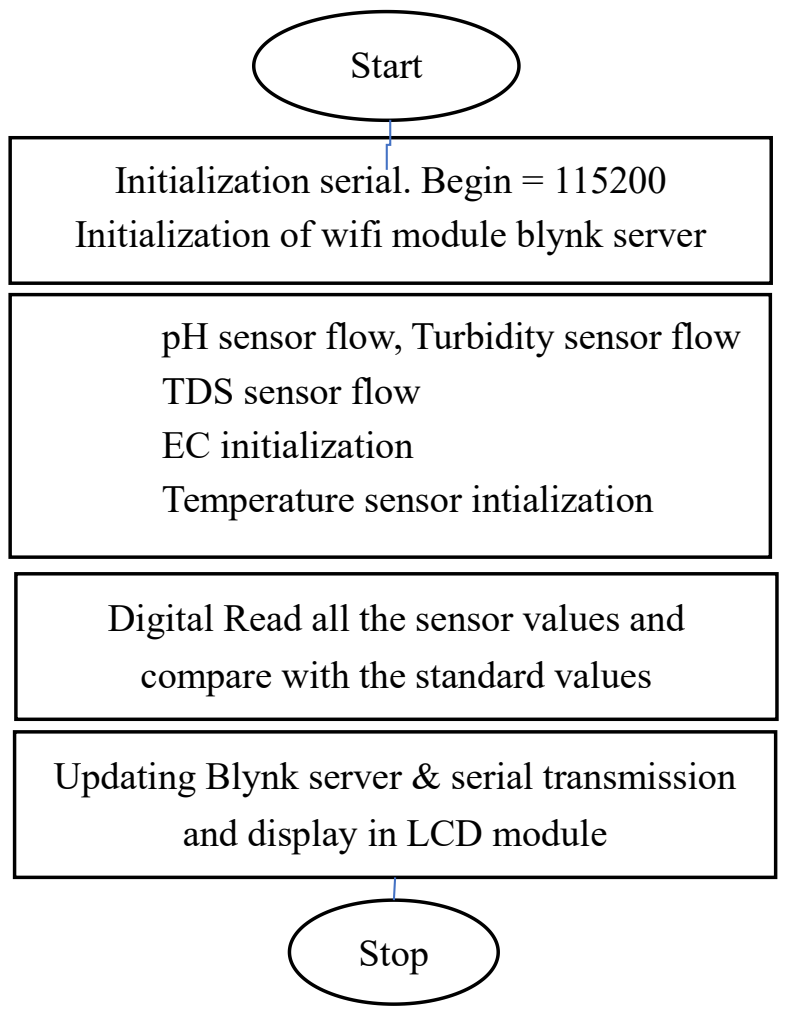

Figure 2.2 Water reservoir monitoring system software flow diagram

\section{Results and discussion}

The smart embedded system board is used for monitoring water and sewage water quality by providing all participants with appropriate and timely data for complete result analysis. Water quality monitoring system delivers authorities for water quality detection and monitoring which is the reliable, autonomous, flexible, ubiquitous, and smart one. Sensor nodes will be placed on all water reservoirs. System-designed executable files will be loaded in a waterboard and users for quality checking. This system has many advantages like portability, low cost, vast area coverage, low power, instantaneous data acquisition, and data classification capability. It will replace the existing manual quality maintenance system. It will enhance the smart city plan. Easy water quality monitoring. Figure 3.1 shows the output displayed in the LCD module. The value displayed is $\mathrm{pH}$ as 7.3, Electrical 
Conductivity as $1489 \mathrm{um}$, and total dissolved solvents as $749 \mathrm{ppm}$. This value is checked for the standard value range and recommended for further processing if needed.

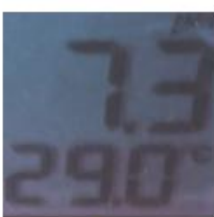

$\mathrm{pH}$

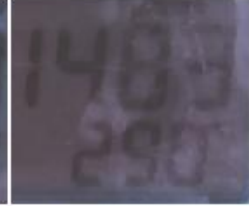

EC

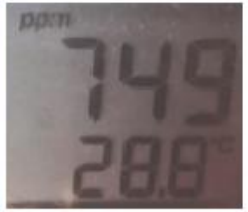

TDS

Figure 3.1 LCD output display physical parameters of water

Table 3.1 shows the table of water physical properties measured by the board and values stored in the database for further processing. The date, time with physical parameters of the water data are stored in the table form and processed for quality checking.

Table 3.1. physical parameter measurement data base values

$\begin{array}{llrlrr}\text { Date } & \text { Time } & \mathrm{pH} & \begin{array}{l}\text { TDS } \\ \text { (ppm) }\end{array} & \begin{array}{l}\text { Temp } \\ \text { (Celsius) }\end{array} & \begin{array}{l}\text { EC } \\ \text { (us } / \mathrm{cm})\end{array} \\ 12.2 .2021 & 9.00 \mathrm{am} & 7.1 & 1105 & 30 & 2020 \\ 3.3 .2021 & 9.00 \mathrm{am} & 6.9 & 1110 & 29.7 & 2000 \\ 5.4 .2021 & 11.00 \mathrm{am} & 6.8 & 1042 & 32 & 2000 \\ 8.5 .2021 & 2.00 \mathrm{pm} & 7 & 1230 & 31 & 2300\end{array}$

The values measured by the sensor are sent to the cloud for storing and displaying information. The sensor board and the sensor are shown in figure 3.2. The input sent from the sensor is processed by the microcontroller and through the communication module, it is sent to the cloud for display.

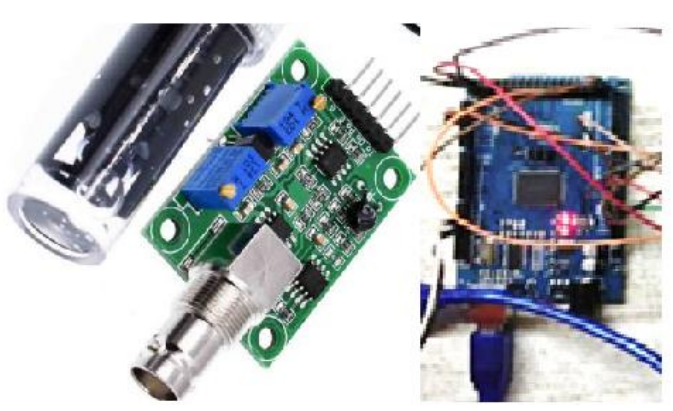

Figure $3.2 \mathrm{pH}$ sensor and board to send information

Figure 3.3 shows the output of the Blynk cloud and displays it in graphical form on the mobile app.

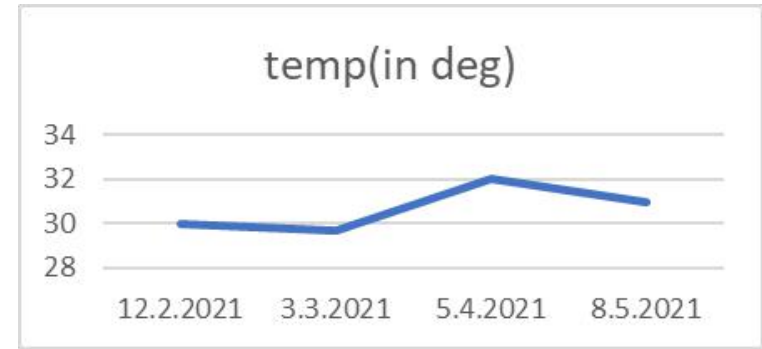

Figure. 3.3 (a)Temperature in graphical form

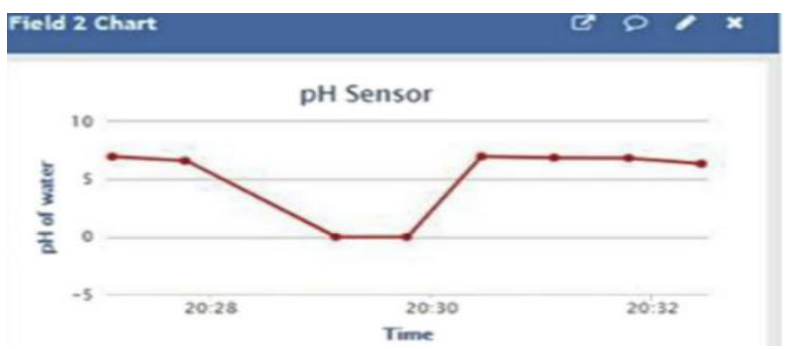

(b) $\mathrm{pH}$ sensor value in mobile app 

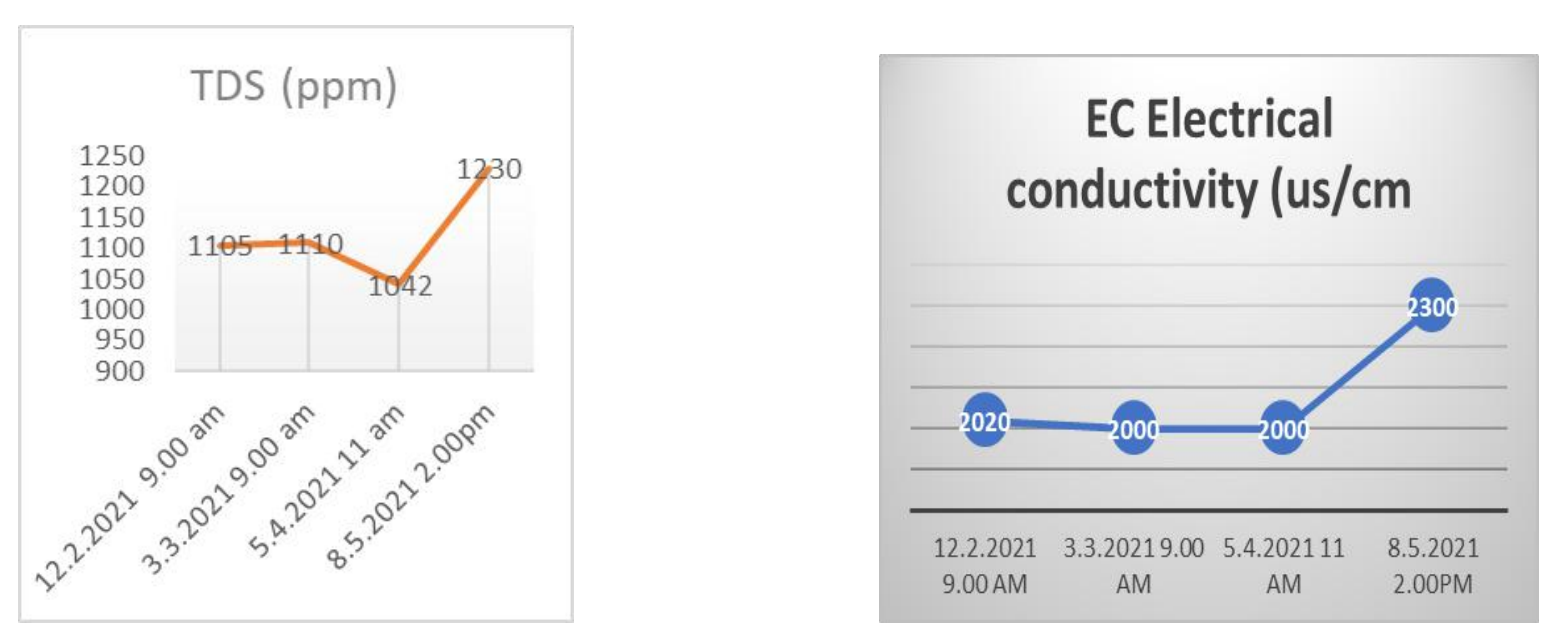

Figure 3.3 (c ) TDS sensor value in PC display

(d) EC value output in database

Figure 3.3. Display of measured information in graphical form Blynk mobile app and PC.

The PC displays the temperature in graphical form as shown in figure 3.3 (a). The Cloud platform displays the potential of Hydrogen $(\mathrm{pH})$ information as shown in figure 3.3 (b). The Total dissolved solvents and electrical conductivity stored in the database are displayed at the PC as shown in figure 3.3 (c) and (d). The same information is sent anywhere in real-time for checking purposes through the cloud platform.

\section{Conclusion}

This system is designed with less cost. The water board could able to afford this board at a reasonable cost. The system is designed for efficiency. The working is checked with the standard value of $\mathrm{pH}$ value. Hardware modules and software modules for performing physical characteristics of water are constructed. In this designed system, the monitoring system is interfaced with sensors such as the potential of hydrogen $(\mathrm{pH})$, temperature, humidity, electrical conductivity, turbidity, Total Dissolved Solvents (TDS) with the microcontroller unit along with the communication module. The collected data is sent to the blynk cloud platform. All information is visible to the end-user at the instant of measurement for checking the quality of water. If the physical parameters are not within the standard range, the water is recommended to check its biochemical parameters at the laboratory for further quality identification. This system integrates IoT and WSN technology for water reservoir monitoring systems. The quality of drinking water is maintained in real-time.

The system effectiveness, the buoy's steadiness in severe environmental conditions, the energy consumption of the system, data communication effectiveness, efficiency, reliability are checked. The web-based display information is evaluated. Physical properties of water are checked to maintain standard and quality automatically. The system may be extended to do sewage water treatment plants.

\section{References}

1. https://www.who.int/water_sanitation_health/

2. Sung Min Cha, Seo Jin Ki, Kyung Hwa, Joon Ha Kim, “ Effect of environmental flow management on river water quality: A case study at Yongsan river, Korea”, February 2009, water science \& technology, vol. 59 (12), pp.2437-46.

3. Jyotirmaya Ijaradar and Subhasish Chatterjee, "Real-Time Water Quality Monitoring System” International 
Research Journal of Engineering and Technology (IRJET) e-ISSN: 2395-0056 Volume: 05 Issue: 03, Mar-2018, pp.1166 -1170.

4. Sridharan, S" Water Quality Monitoring System Using Wireless Sensor Network", International Journal of Electronic Communications Engineering Advanced Research, 2014, Vol.3, 399-402.

5. Natalia Szczerbinska, Malgorzata Galczynska, "Biological methods used to assess surface water quality", Arch.Pol.Fish., 2015 vol. 23, pp.185-196.

6. Dr. Seema Tiwari, “Water quality parameters - A review”, International Journal of Engineering Science Invention Research \& Development, Vol. I, Issue IX, March 2015, pp.319-324.

7. Singh R, Gehlot A, Kaushik M, "Dynamic Modeling and Decision System for Smart Bin in Cities for Waste Management", International Journal of Intelligent Communication, Computing, and Networks, Vol 1, issue 1, PP 001-009, Aug 2020.

8. Rogers R, "Communication Protocols for Smart Sensors in IoT Applications", International Journal of Intelligent Communication, Computing, and Networks, Vol 1, issue 2, PP, Jan 2021.

9. Singh A, Sharma PK, Dwivedi S, "Digital Temperature Scanner Using IOT" International Journal of Intelligent Communication, Computing, and Networks, Vol 2, issue 2, PP 26-30, July 2021.

10. Thakur R K, Internet of Things (IoT) Security: Present Status and Future Direction, " International Journal of Intelligent Communication, Computing, and Networks, Vol 2, issue 2, PP 36-42, July 2021. 